Rev. Elev. Méd. vét. Pays trop., 1977, 30 (2) : 143-148.

\title{
Sérologie de la péripneumonie Utilisation du matériel «Microtiter» dans une microréaction de fixation du complément selon la méthode de Kolmer
}

\author{
par M. P. DOUTRE $(*)$ et B. TOURE $(* *)$
}

\begin{abstract}
RÉSUMÉ
Le matérıel « Microtiter » est employé dans la réalisation d'une microréaction de fixation du complément. Les différents temps de la méthode font l'objet d'une description détaillée.

Cette microtechnique, sensible et fidèle, très standardisée, d'exécution rapide et aisée, permet de traiter un nombre important de sérums en utilisant de petites quantités de réactifs. Son utilisation est néanmoins limitée au cadre d'un laboratoire normalement équipé.
\end{abstract}

La sérologie de la péripneumonie bovine, tout particulièrement en Australie et en Afrique tropicale, a donné lieu à maints développements au cours des récentes décennies. Les méthodes présentées par de nombreux auteurs ont toutes contribué à faciliter le diagnostic de la maladie et à mieux connaître la cinétique des anticorps sériques ou le devenir des substances antigéniques élaborées pendant l'affection. Dans cet ensemble, la fixation du complément (FC) demeure le test le plus communément pratiqué lorsqu'il est impératif de préciser l'extension d'un foyer, de suivre le déroulement d'une épreuve d'immunité, etc...

Les plaques en plexiglas type O. M. S. (80 cupules) ont été utilisées par PERREAU, dans une réaction d'hémagglutination indirecte (7). par PROVOST en fixation du complément selon CAMPBELL et TURNER $(9,10)$ et par KARST (5). Actuellement, les microplaques (96 cupules) retiennent le choix. PEARSON et

(*) Chef du Service de Bactériologie. Laboratoire national de l'Elevage et de Recherches vétérınaires (I. S. R. A.), B. P. 2057, Dakar Hann, Sénégal.

(**) Technicien supérieur. Laboratoire National de l'Elevage et de Recherches Vétérinaires (I. S. R. A.).
McPHERSON (6) et PERREAU (8) les emploient dans une microréaction de $\mathrm{FC}$ selon CAMPBELL et TURNER, ainsi que SCUDAMORE (12) qui utilise une microréaction de FC à chaud nécessitant une centrifugation avant lecture.

L'emploi du matériel « Microtiter » a connu ces dernières années une large diffusion en sérologie virale et bactérienne, humaine ou animale $(2,3,11)$. Le texte qui suit présente une technique de microréaction de $\mathrm{FC}$ à froid, dérivée de la méthode classique de KOLMER, exécutée avec ce matériel.

\section{MATÉRIEL ET MÉTHODE}

\section{A. MATÉRIEL}

Les accessoires utilisés : microplaques, compte gouttes ( 25 et $50 \mu \mathrm{l})$, adhésif, etc... sont désormais classiques $\left(^{*}\right)$.

(*) Cooke Engineering Company, Medical Research Division, 735 North Saint Asaph Street, Alexandria, Virgınıa 22314. Représenté en France par Poly-Labo, Paul Block et Cie, 305 route de Colmar, 67023 Strasbourg Cedex. 
Deux doivent néanmoins être mentionnés:

- le manche pour micro-dilueurs « Multi»,

- le micro-agitateur AM69 pour 4 plaques : l'agitation manuelle dans un plan horizontal étant insuffisante pour la bonne diffusion des réactifs et du couple hémolytique.

\section{Produits biologiques et réactifs}

- Sérums à analyser : sang recueilli le plus stérilement possible pour diminuer le nombre de sérums anticomplémentaires. Au laboratoire, les sérums sont séparés par centrifugation.

- Tampon véronal-calcium-magnésium

- Antigène : préparé selon la technique de CAMPBELL et TURNER (1). Le surnageant d'un lot ancien est tout particulièrement recommandé.

- Complément : lyophilisé ou frais, mais de bon titre.

- Sérum hémolytique de lapin anti-hématies de mouton.

- Hématies de mouton: le protocole suivant est préconisé (4). Utiliser toujours la même brebis. Avec une séringue de $50 \mathrm{ml}$, contenant $25 \mathrm{ml}$ de solution d'Alsever, récolter stérilement $25 \mathrm{ml}$ de sang; mettre les $50 \mathrm{ml}$ dans un ballon stérile. Attendre au moins 3 jours avant d'utiliser les hématies, les laver alors 3 fois par centrifugations successives en tampon.

On utilise dans la réaction une suspension à environ 3 p. 100 . Pour plus de précision, il est recommandé de standardiser la suspension en procédant aux opérations qui suivent :

- à partir de $5 \mathrm{ml}$ de culot d'hématies, après lavage, préparer $50 \mathrm{ml}$ de suspension d'hématies,

- utiliser le spectrophotomètre (au laboratoire de Dakar : Beckman, model 25, U. S. A.) à $540 \mathrm{~m} \mu$ de longueur d'onde, étalonner le $\mathrm{O}$ avec une solution de carbonate de sodium $\left(\mathrm{Na}_{2} \mathrm{CO}_{3}\right)$ au 1 p. 1000 ,

- mesurer le pouvoir d'absorption :

suspension d'hématies : $0,1 \mathrm{ml}$, solution de $\mathrm{Na}_{2} \mathrm{CO}_{3}: 4,8 \mathrm{ml}$.

L'absorption finale doit être de $135 \pm 5$.

Le volume $\mathrm{V}$ de la suspension d'hématies à préparer pour obtenir 135 est ainsi calculé :

$V=\frac{\text { absorption lue }}{135} \times \underset{\text { à titrer. }}{\text { volume de la suspension }}$
Lorsque la suspension définitive d'hématies est obtenue, vérifier que :

suspension définitive d'hématies : $0,1 \mathrm{ml}$,

solution $\mathrm{Na}_{2} \mathrm{CO}_{3} \quad: 4,8 \mathrm{ml}$.

ont un pouvoir d'absorption de $135 \pm 5$. Faire 2 lectures.

Conservation au frigidaire de cette suspension d'hématies.

\section{B. MÉTHODE}

La disposition des plaques de microtitration est qualifiée : d' « horizontale » lorsque la longueur est placée de gauche à droite de l'opérateur, de "verticale » lorsque la largeur occupe cette situation.

\section{1) Titrage du système hémolytique}

Le titrage d'un sérum hémolytique s'est révélé très difficile à réaliser en microtiter. Après différents essais, le sérum hémolytique est employé à la dilution de 1 p. 1000 pour une suspension d'hématies de mouton présentant un pouvoir d'absorption de $135 \pm 5$. La sensibilisation des hématies est effectuée en mélangeant à volumes égaux sérum hémolytique à 1 p. 1000 et suspension d'hématies à $135 \pm 5$ d'absorption. Dans toutes les réactions le couple hémolytique est ajouté sous un volume de $25 \mu \mathrm{l}$.

\section{2) Titrage du complément}

Préparer sous le plus petit volume nécessaire et suffisant (économie du réactif) une solution au $1 / 15$, au $1 / 20$ et au $1 / 25$ du complément.

\section{Dilution du complément}

La plaque est tenue "verticale ».

- Partager la plaque en 3 zones en traçant au crayon feutre 2 lignes entre les rangées 4 et 5 et 8 et 9 (fig. 1).

- Distribuer $50 \mu \mathrm{l}$ de tampon dans toutes les cupules, sauf celles de la rangée « verticale » $\mathbf{H}$.

- Distribuer $50 \mu \mathrm{l}$ de complément dans les 2 premières rangées « verticales » $\mathrm{H}$ et $\mathrm{G}$ :

au $1 / 15$ dans les cupules $\mathrm{G} 1$ à $\mathrm{G} 4$ et $\mathrm{H} 1$ à $\mathrm{H} 4$,

au 1/20 dans les cupules $\mathrm{G} 5$ à $\mathrm{G} 8$ et $\mathrm{H} 5$ à $\mathrm{H} 8$. $\mathrm{H} 12$, au $1 / 25$ dans les cupules $G 9$ à $\mathrm{G} 12$ et $\mathrm{H} 9$ à 


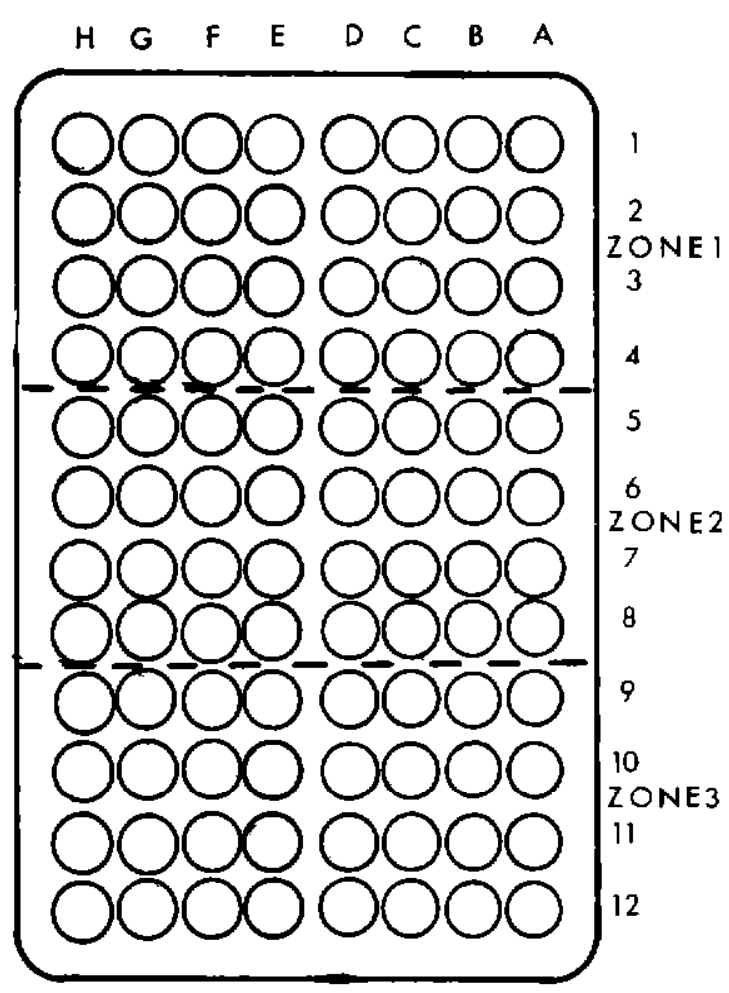

Fig. 1. - Dilution du complément pour son titrage.

- Diluer avec les microdilueurs de $50 \mu \mathrm{l}$, montés sur le manche, de la rangée $G$ à la rangée $A$, en passant par les rangées « verticales » intermédiaires $F, E, D$...A.

Ainsi de gauche à droite, dans la zone I, le complément se trouve réparti à des dilutions multiples du $1 / 15$, sous un volume de $50 \mu \mathrm{l}$, à raison de 4 cupules pour chaque dilution; dans la zone 2 , à des dilutions multiples du $1 / 20$; dans la zone 3 , à des dilutions multiples du 1/25.

Titrage (fig. 2)

- Ajouter dans chaque cupule :

$25 \mu l$ de tampon,

$25 \mu$ d'antigène titré (1/20, voir plus lo1n).

- Recouvrir la plaque d'adhésif auto-collant.

- Placer quelques secondes sur le vibreur.

- Incuber $1 \mathrm{~h}$ à $37^{\circ} \mathrm{C}$ au bain-marie.

- Ajouter $25 \mu$ de système hémolytique par cupule.

- Recouvrir la plaque d'adhésif au-collant.

- Placer quelques secondes sur le vibreur.

- Incuber $1 \mathrm{~h}$ à $37^{\circ} \mathrm{C}$ au bain-marie.

- Lire 2 à $3 \mathrm{~h}$ après au miroir de lecture.

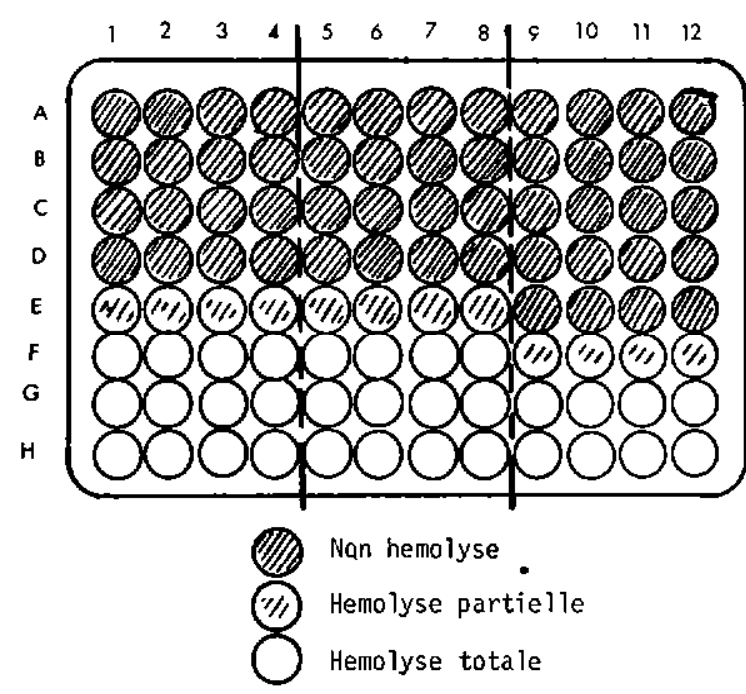

Fig. 2. - Titrage du complément.

Le titre du complément correspond à la dilution maximale dans les 4 cupules où l'hémolyse est totale (ex. : titre au 1/80: cupules $\mathrm{F} 5$ à $\mathrm{F} 8$; au $1 / 100: \mathrm{F} 9$ à $\mathrm{F} 12$; au $1 / 60: \mathrm{F} 1$ à $F 4$, etc...). On utilise, pour la réaction, le complément à 2 unités : Exemple : si le titre est le $1 / 80$, utiliser le $1 / 40$.

\section{3) Titrage de l'antigène (fig. 3)}

La plaque est tenue de façon " horizontale ».

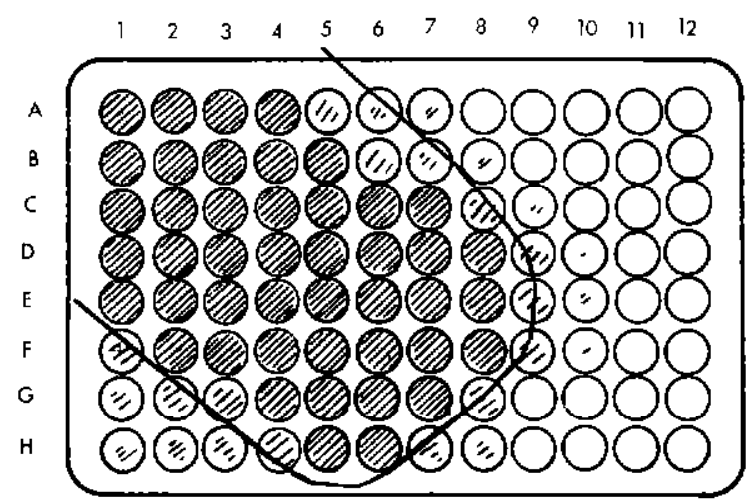

Fig. 3. - Titrage de l'antigène (voir texte).

Disposer d'un sérum étalon décomplémenté de titre connu (ex. 1/640). Préparer la dilution au $1 / 5$ de ce sérum.

- Distribuer $25 \mu$ de tampon dans toutes les cupules, sauf celles de la rangée « verticale » 1 .

- Distribuer $25 \mu$ lde sérum étalon au $1 / 5$ dans les deux premières rangées "verticales " 1 et 2 . 
- Diluer avec 8 microdilueurs de $25 \mu 1$, montés sur le manche, de la rangée « verticale » 2 à la rangée 12 , en passant par les rangées « verticales » intermédiaires 3 à 12 .

Préparer 8 dilutions d'antigène situées de part et d'autre d'une dilution optimale, ex : $1 / 5,1 / 10,1 / 20 \ldots 1 / 640$.

- Répartir $25 \mu$ par cupule de l'antigène au 1/640 dans la rangée " horizontale » $\mathrm{H} ; 25 \mu \mathrm{l}$ par cupule de l'antigène au 1/320 dans la rangée " horizontale » $\mathrm{G}$, etc... $25 \mu \mathrm{l}$ par cupule de l'antigène au 1/5 dans la rangée "horizontale" $A$, - Ajouter le complément à un titre moyen (2 U) sous un volume de $50 \mu \mathrm{l}$ par cupule. lant.

- Recouvrir la plaque d'adhésif auto-col-

- Placer quelques secondes sur le vibreur.

- Incuber $1 \mathrm{~h}$ à $37^{\circ} \mathrm{C}$ au bain-marie.

- Ajouter $25 \mu 1$ de système hémolytique par cupule etc...; comme ci-dessus: adhésif, vibreur, incubation $1 \mathrm{~h}$ à $37^{\circ} \mathrm{C}$, lecture 2 à $3 \mathrm{~h}$ plus tard.

Le titre de l'antigène correspond à la plus forte dilution donnant le titre exact du sérum étalon, sans hémolyse dans les premières cupules de la rangée horizontale.

La figure 3 représente un titrage d'antigène réalisé comme ci-dessus. Les rangées $\mathrm{A}$ et $\mathrm{B}$ révèlent un excès d'antigène, les rangées $D, E$, $\mathrm{F}$, $\mathrm{G}$ un manque croissant, avec hémolyse dans les premières cupules. Le titre à retenir correspond à la ligne $\mathrm{D}$, soit la dilution du 1/20. L'antigène utilisé à une dilution supérieure dans la réalisation de la réaction entraînerait la formation d'un faux phénomène de zone pour les basses dilutions du sérum. La courbe classique a été surimposée sur la figure. L'utilisation de dilutions plus serrées de l'antigène, trop proches du $1 / 20$, n'aurait pas permis la mise en évidence des différentes zones (excès, quantité optimale manque d'antigène).

\section{4) Exécution de la réaction}

Deux solutions sont proposées :

- exécution de la réaction avec un seul témoin sérum (à la dilution du 1/10). Dans ce cas, la recherche des anticorps se poursuit jusqu'à la dilution $1 / 5120$;

- exécution de la réaction avec un témoin sérum pour chaque dilution. Dans ce cas, la recherche des anticorps ne dépasse pas la dilution du 1/160 (à moins que l'on accepte de placer des plaques bout à bout).

En effet, en péripneumonie bovine et dans les conditions de récolte des prélèvements en brousse il arrive souvent qu'un sérum, anti-complémentaire aux basses dilutions, soit positif et non anticomplémentaire aux dilutions supérieures.

a) Exécution de la réaction avec un seul témoin sérum (à la dilution du 1/10) (fig. 4).

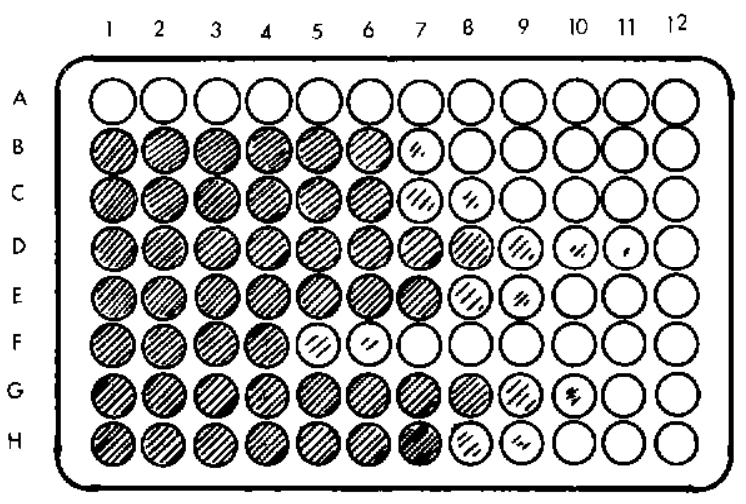

Fig. 4. - Titrage de différents sérums avec témoin sérum à la dilution du $1 / 10$ (ligne $\mathrm{A}$ : sérum négatif).

- Diluer les sérums au $1 / 5$ en tampon et décomplémenter cette dilution $(30 \mathrm{mn}$ à 58 $60^{\circ} \mathrm{C}$ ).

- La plaque est tenue de façon « horizontale " (les sérums sont supposés offrir un titre élevé, 7 sérums +1 sérum positif de référence sont étudiés par plaque, jusqu'à la dilution du $1 / 5120$; dans le cas contraire, la plaque peut être tenue «verticale » et permet de traiter 11 sérums +1 sérum positif de référence, jusqu'à la dilution du $1 / 320$.

- Distribuer (à l'aide des compte-gouttes en plastique à usage unique), pour chaque sérum, $25 \mu \mathrm{l}$ de la dilution au 1/5 dans la première cupule (ex. : A1), dans la seconde cupule (A2) et dans la dernière (A12) (témoin sérum anticomplémentaire) d'une même rangée « horizontale "; le second sérum se place en B1, B2, B12, etc...

- Ne pas oublier le ou les sérums de référence.

- Ajouter $25 \mu \mathrm{l}$ de tampon dans chaque cupule, sauf celles de la rangée "verticale » 1 .

- Diluer avec 8 microdilueurs de $25 \mu 1$, montés sur manche, de la rangée "verticale » 2 à la rangée 11 , en passant par les rangées « verti- 
cales " intermédiaires 3 à 11 (il est évident que si la plaque avait été tenue "verticale », $12 \mathrm{mi}-$ crodilueurs auraient été employés, de la rangée " verticale * $G$ à la rangée $B$, la rangée $A$ contenant les témoins sérum).

- Ajouter $25 \mu \mathrm{l}$ d'antigène titré $(1 / 20)$ dans toutes les cupules, sauf celles de la dernière rangée « verticale " 12.

- Distribuer $50 \mu \mathrm{l}$ de complément au titre choisi (2U) dans toutes les cupules. Cette opération doit être faite en fin de journée ouvrable, le complément libre risquant d'être détruit le lendemain matin, malgré la mise à $+4^{\circ} \mathrm{C}$, s'il a été réparti trop tôt.

Etc... adhésif, vibreur, incubation une nuit à $+4^{\circ} \mathrm{C}$, système hémolytique, adhésif, vibreur, incubation $30 \mathrm{mn}$ au bain-marie à $37^{\circ} \mathrm{C}$, la lecture 2 à $3 \mathrm{~h}$ plus tard. Bien observer le comportement des témoins sérums au 1/10 (rangée « verticale »12).

b) Exécution de la réaction avec un témoin sérum pour chaque dilution (fig. 5).

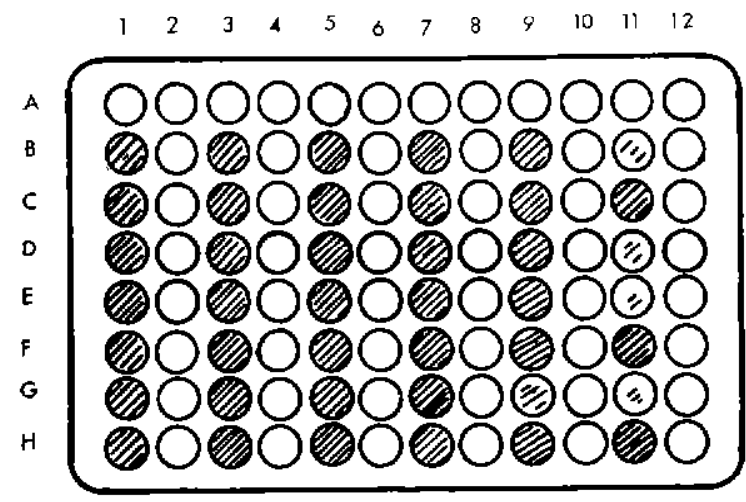

Fig. 5. - Titrage de différents sérums avec témoın sérum pour chaque dilution (ligne $\mathrm{A}$ : sérum négatif).

Dans ce cas, la plaque est tenue obligatoirement « horizontale ».

- Distribuer, pour chaque sérum, $25 \mu \mathrm{l}$ de la dilution au 1/5 dans les 4 premières cupules d'une même rangée horizontale (ex. : $\mathrm{A} 1, \mathrm{~A} 2$, $\mathrm{A} 3, \mathrm{~A} 4)$; le second sérum se place en $\mathrm{B} 1, \mathrm{~B} 2$, B3, B4, etc...

- Ne pas oublier le ou les sérums de référence,

- Ajouter $25 \mu$ de tampon dans chaque cupule, sauf celles de la rangée "verticale " 1 et 2.

- Diluer avec 8 microdilueurs de $25 \mu l$, montés sur manche, de la rangée "verticale" 3 à la rangée 11, en passant par les rangées inter- médiaires $5,7,9$. Rincer et sécher à la flamme les microdilueurs, puis effectuer une nouvelle série de dilutions de la rangée 4 à la rangée 12, en passant par les rangées intermédiaires 6, 8, 10.

- Ajouter $25 \mu \mathrm{l}$ d'antigène au titre choisi dans toutes les cupules des rangées "verticales» impaires et $25 \mu \mathrm{l}$ de tampon dans celles des rangées paires.

Etc... La suite des manipulations comme ci-dessus (a).

\section{INTERPRÉTATION DES RÉSULTATS}

L'interprétation des résultats est identique à celle déjà connue de la fixation du complément en tubes, selon KOLMER, à savoir : est considéré comme positif tout sérum ++++ au $1 / 80$, et douteux, tout sérum ++++ au $1 / 40$.

\section{DISCUSSION}

Les avantages de la microréaction de fixation du complément, dérivée de la méthode de KOLMER, sur la technique classique en tubes sont les suivants :

- Economie des réactifs utilisés. En péripneumonie bovine, ces derniers sont généralement d'un prix de revient faible; néanmoins dans les pays tropicaux, le titre du complément lyophilisé subit très souvent une forte chute pendant le transport par voie aérienne (élévation de température) en dépit des précautions prises, aussi peut-on rechercher à économiser un complément de haut titre.

- Rapidité d'exécution des manipulations, grâce à la réalisation semi-automatique des dilutions par l'emploi des microdilueurs montés sur manche, d'où possibilité de traiter en un temps minimal un nombre de sérums élevé. L'exécution de la réaction, telle qu'elle vient d'être décrite, concerne des sérums titrés pour un ensemble de dilutions croissantes, jusqu'à la limite de positivité et au-delà. Ce type de tirage est valable lorsque l'on tient à connaître le taux d'anticorps au cours d'une expérimentation. Lorsque l'on s'attache uniquement à déceler la maladie dans un groupe d'animaux, les plaques peuvent être fractionnées selon la volonté de l'opérateur et seules quelques dilutions bien déterminées $(1 / 40,1 / 80)$ peuvent être retenues.

Les manipulations sont faciles à réaliser et un technicien ordinaire acquiert très rapidement 
le « coup de main » que réclame le matériel « microtiter» (micropipettes, microdilueurs, etc.). L'utilisation des divers accessoires et leur entretien obéissent à des règles strictes, annoncées par le fabricant, qui doivent être impérativement respectées (microdilueurs).

Le complément titré doit être réparti en fin de journée ouvrable, comme il a été dit ci-dessus. Si cette condition est observée, un nombre important de plaques peut être utilisé au cours de la même séance. La fixation à froid peut être réalisée dans un frigidaire ordinaire, avec un encombrement minimal, les plaques s'empilant aisément les unes sur les autres grâce à un système de tétons. La cellophane adhésive, qui obture de façon étanche les cupules, évite toute dessiccation possible. Le miroir grossissant rend la lecture facile avec un peu d'habitude.

- La standardisation diminue les risques d'erreur et la méthode est sensible et fidèle.

$\mathrm{Au}$ chapitre des inconvénients, le coût du matériel ne doit pas être retenu. L'amortissement étant assuré par la multiplicité des usages auxquels il se prête. Par contre, au passif, on doit reconnaître que ce type de microréaction réclame, pour son exécution, le confort, le calme et l'équipement général d'un laboratoire, aussi ne peut-elle être accomplie sur le terrain. De même doit-on noter la nécessité de disposer d'un complément d'excellent titre, comme dans la réaction classique de CAMPBELL et TURNER.

\section{SUMMARY}

Serology of contagious bovine pleuropneumonia

a Kolmer microtiter complement fixation test

«Microtiter» system is employed in a complement fixation microtechnique. The various phases of the technical process are described in details. This sensitive and constant microtechnique can be easily performed and allows serial serological diagnosis with saving of biological reagents. Its use is restricted to a normally equipped laboratory.

\section{RESUMEN \\ Serologia de la perineumonia. \\ Utilización del material « Microtiter » para una microreacción de fijación del complemento según el método de Kolmer}

Se utiliza el material «Microtiter » para realizar una microreacción de fijación del complemento. Se describen en detalle las diferentes fases del método.

Dicha microtécnica, sensible y fiel, muy unificada, de ejecución rápida y fácil, permite el tratamiento de un gran número de sueros al utilizar pequeñas cantidades de reactivos. Sın embargo, no puede ser utilizada más que en un laboratorio normalmente equipado.

\section{BIBLIOGRAPHIE}

1. CAMPBELL (A. D.), TURNER (A. W.). Studies on contagious pleuropneumonia of cattle. An improved complement-fixation test. Aust. vet. J., 1953, 29 (6) : 154-163.

2. CONRATH (T. B.). Handbook of microtiter procedures. Cambridge, Mass., Dynatech Corpor., $1972,475 \mathrm{p}$.

3. ELLIS (E. M.). Microtiter serologic techniques for diagnostic bacteriology. Proc. 77th annual Meet. U.S. anim. Hlth Ass., 1973 (publié 1974) 77 : 563-581.

4. FENSTERBANK (R.). - Com. person., 1975.

5. KARST (O.). Contagious bovine pleuropneumonia : a plate complement fixation test employed at the Federal Department of Veterinary Research, Vom. Bull. epiz. Dis. Afr., 1970, 18 (1) : 5-11.

6. PEARSON (C. W.), McPHEARSON (G.). A modified complement fixation test for contagious bovine pleuropneumonia. Aust. vet. J., 1966, 42 (9) : 324-327.

7. PERREAU (P.), PROVOST (A.), REGNOULT (R.), ORUE (J.). Valeur de la réaction d'hémagglutination indirecte dans la péripneumonie bovine. Rev. Elev. Méd. vét. Pays trop., 1964, 17 (1) : 5-14.
8. PERREAU (P.). Le diagnostic sérologique de la pérıpneumonie. Progrès techniques actuels. Bull. Off. int. Epizoot., 1975, 84 : 349-358.

9. PROVOST (A.). Adaptation sur plaques de la réaction de fixation du complément pour la péripneumonie : application à la décentralisation du diagnostic. - Rapport à la IVe réunion groupe Experts F.A.O./O.I.E./O. U.A. sur la péripneumonie bovine contagieuse, Paris, 15-20 mars 1971. Bull. Epiz. Afr., 1972 (n ${ }^{\circ}$ sp. juin) : 13-22.

10. PROVOST (A.). Recherches immunologiques sur la péripneumonie. XIV. Description de deux techniques applicables sur le terrain pour le diagnostic de la maladie. Rev. Elev. Méd. vét. Pays trop., 1972,25 (4) : 475-496.

11. RENOUX (G.), PLOMMET (M.), PHILIPPON (A.). Microréactions d'agglutination et de fixation du complément pour le diagnostic des brucelloses. Ann. Rech. vét., 1971, 2 (2) : 263-269.

12. SCUDAMORE (J. M.). Evaluation of the field C. F. T. in the diagnostic and control of C. B. P. P. Trop. anim. Hlth Prod., 1975, 7 (2) : 73-79. 